\title{
Synthetic Chemical Inducers and Genetic Decoupling Enable Orthogonal Control of the rhaBAD Promoter
}

Ciarán L. Kelly, ${ }^{\dagger}$ Zilei Liu, $^{\ddagger}$ Akihide Yoshihara, ${ }^{*},{ }^{\S}$ Sarah F. Jenkinson, ${ }^{\ddagger}$ Mark R. Wormald, ${ }^{\Perp}$ Jose Otero, ${ }^{\perp}$ Amalia Estévez, ${ }^{\perp}$ Atsushi Kato, ${ }^{\#}$ Mikkel H. S. Marqvorsen, ${ }^{\ddagger}$ George W. J. Fleet, ${ }^{\ddagger}$ Ramón J. Estévez, ${ }^{\perp}$ Ken Izumori, ${ }^{\S}$ and John T. Heap ${ }^{* \dagger}$

${ }^{\dagger}$ Centre for Synthetic Biology and Innovation, Department of Life Sciences, Imperial College London, South Kensington Campus, London SW7 2AZ, U.K.

${ }^{\ddagger}$ Chemistry Research Laboratory, Department of Chemistry, University of Oxford, Oxford, OX1 3TA, U.K.

${ }^{\S}$ International Institute of Rare Sugar Research and Education, Kagawa University, Miki, Kagawa 761-0795, Japan

"Glycobiology Institute, Department of Biochemistry, University of Oxford, Oxford, OX1 3QU, U.K.

${ }^{\perp}$ Departamento de Química Orgánica and Centro Singular de Investigación en Química Biolóxica e Materiais Moleculares, Universidade de Santiago de Compostela, 15782 Santiago de Compostela, Spain

\#Department of Hospital Pharmacy, University of Toyama, Toyama 930-0194, Japan

Supporting Information

ABSTRACT: External control of gene expression is crucial in synthetic biology and biotechnology research and applications, and is commonly achieved using inducible promoter systems. The E. coli rhamnose-inducible $r h a B A D$ promoter has properties superior to more commonly used inducible expression systems, but is marred by transient expression caused by degradation of the native inducer, L-rhamnose. To address this problem, 35 analogues of Lrhamnose were screened for induction of the $r h a B A D$ promoter, but no strong inducers were identified. In the native configuration, an inducer must bind and activate two transcriptional activators, RhaR and RhaS. Therefore, the expression system was
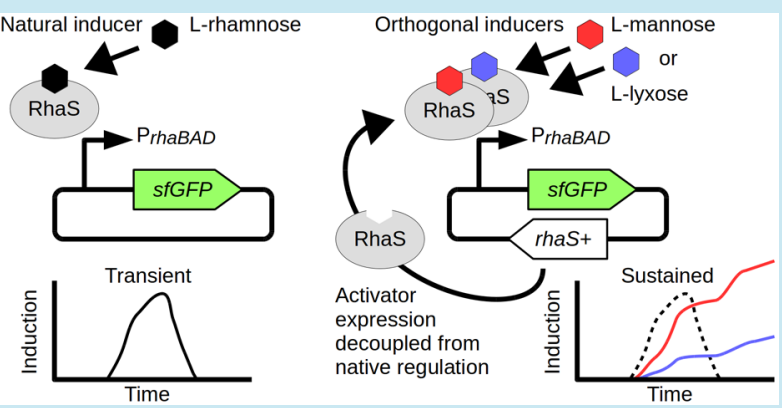
reconfigured to decouple the rhaBAD promoter from the native rhaSR regulatory cascade so that candidate inducers need only activate the terminal transcription factor RhaS. Rescreening the 35 compounds using the modified rhaBAD expression system revealed several promising inducers. These were characterized further to determine the strength, kinetics, and concentration-dependence of induction; whether the inducer was used as a carbon source by E. coli; and the modality (distribution) of induction among populations of cells. L-Mannose was found to be the most useful orthogonal inducer, providing an even greater range of induction than the native inducer L-rhamnose, and crucially, allowing sustained induction instead of transient induction. These findings address the key limitation of the rhaBAD expression system and suggest it may now be the most suitable system for many applications.

KEYWORDS: rhaBAD, inducible promoter, expression system, rhamnose, mannose, nonmetabolized inducer

$\mathrm{E}$ xperimental control of gene expression is a crucial requirement in synthetic biology and biotechnology research and applications and in many studies of natural biological systems. Inducible promoter systems are commonly used to achieve this external control through initiation of transcription. Promoters have been identified that respond to a diverse array of stimuli, including sugars, ${ }^{1-3}$ antibiotics, ${ }^{4}$ metals, ${ }^{5,6}$ quorum sensing signaling molecules, ${ }^{7-9}$ temperature, ${ }^{10}$ and many others. The L-rhamnose-inducible promoter of the rhaBAD operon $\left(\mathrm{P}_{\text {rhaBAD }}\right)$ of Escherichia coli (E. coli) has been used extensively to control expression of many heterologous genes in E. coli and other bacteria. ${ }^{11-20} \mathrm{P}_{\text {rhaBAD }}$ is a member of the AraC-XylS family of positively regulated expression systems ${ }^{21,22}$ and has several advantages over other inducible promoter systems such as the $\mathrm{P}_{\text {tet }}$ (tetracycline), $\mathrm{P}_{\text {lac }}$ (lactose) or $\mathrm{P}_{\text {araBAD }}$ (arabinose) inducible systems and their derivatives. $\mathrm{P}_{\text {rhaBAD }}$ is capable of very high levels of expression, ${ }^{23}$ but unlike $\mathrm{P}_{\text {lac }}, \mathrm{P}_{\text {araBAD }}$, or $\mathrm{P}_{t e t}$ displays undetectable baseline gene expression in the absence of inducer. ${ }^{17,24}$ This level of control is useful generally, but particularly for the expression of genes encoding toxic products, where "leaky" expression can lead to cellular toxicity, difficulties in generating expression constructs, and selective pressure for mutation of the expressed

Special Issue: Synthetic Biology in Europe

Received: January 29, 2016

Published: May 31, 2016 
gene. ${ }^{16}$ Expression from the rhaBAD promoter is linear with respect to inducer concentration, in contrast to the "all or nothing" response of the $\mathrm{P}_{\text {lac }}$ and $\mathrm{P}_{\text {araBAD }}$ systems. ${ }^{16,23} \mathrm{~L}$ Rhamnose, a naturally occurring deoxy-hexose, is nontoxic to bacterial cells, unlike the inducers of some other systems such as tetracycline, the lactose analogue isopropyl $\beta$-D-1-thiogalactopyranoside (IPTG) $)^{23,25-27}$ and in certain cases, arabinose. ${ }^{28}$

In $E$. coli the genes encoding the enzymes required for Lrhamnose metabolism (rhaBAD) and the regulatory proteins required for controlled expression of these genes (rhaSR) are found in two divergent operons. ${ }^{24,29,30}$ The gene encoding the rhamnose-proton symporter $(r h a T)$ is found at another locus. Expression of rhaBAD and rhaT both result from a regulatory cascade. ${ }^{31}$ Transcription of rhaS and rhaR is driven by the AraC/XylS family transcriptional activator RhaR, which is constitutively expressed at a low basal level. ${ }^{17}$ The binding of Lrhamnose to RhaR results in autoinduction of rhaSR expression to high levels. ${ }^{24,31,32}$ In the presence of L-rhamnose, RhaS binds upstream of the RNA polymerase-binding sites of $r h a B A D^{24}$ and $\mathrm{rhaT}^{33}$ leading to high levels of transcription of the downstream genes. ${ }^{34}$ Expression of these genes is subject to catabolite repression, with a CRP-binding site located upstream of the RhaS-binding site. ${ }^{34}$ When bound to L-rhamnose, RhaS also negatively autoregulates its own expression. ${ }^{35}$

In the absence of a preferred carbon source, L-rhamnose is readily metabolized by $E$. coli in both aerobic and anaerobic conditions. ${ }^{36}$ The mechanism of L-rhamnose catabolism is well studied and involves uptake of L-rhamnose into the cell by RhaT, an L-rhamnose:proton symporter, ${ }^{37}$ isomerization by Lrhamnose isomerase (RhaA) to L-rhamnulose, ${ }^{29,38}$ phosphorylation of L-rhamnulose by L-rhamnulose kinase (RhaB), ${ }^{39,40}$ and conversion of the resulting L-rhamnulose-1-phosphate to Llactaldehyde and dihydroxyacetone phosphate (DHAP) by rhamnulose-1-phosphate aldolase $(\mathrm{RhaD}) .^{41}$ L-Lactaldehyde can be metabolized further by an assimilatory aerobic pathway, to pyruvate via lactate, or an anaerobic pathway, to propane-12-diol. ${ }^{42,43}$ This efficient metabolism of L-rhamnose gives rise to the one major limitation of $r h a B A D$ as an expression system: a decrease in inducer concentration over time, which causes expression to decrease and ultimately to cease. ${ }^{17}$

The problem of transient expression caused by inducer degradation has been solved for some other promoter systems by using nonmetabolized inducer analogues, which can be considered effectively orthogonal to native physiology. Commonly used examples include the lactose analogue IPTG $^{44,45}$ and the tetracycline analogue anhydrotetracycline (ATC) ${ }^{46}$ Such inducer analogues have proven widely useful in diverse fields of study and application. To date, no such analogue of L-rhamnose has been described for the rhaBAD expression system.

An ideal inducer of rhaBAD would have similar useful properties to L-rhamnose (no or low toxicity, readily soluble, and readily taken up by cells) and would cause the rhaBAD expression system to respond in a similar way (a linear response to inducer concentration between very low basal expression and high maximal expression) but unlike L-rhamnose, would persist in the cell, resulting in sustained rather than transient induction of the rhaBAD promoter. Such a combination of properties might be found in a structural analogue sufficiently similar to Lrhamnose to be recognized by RhaS and RhaR, but which is a poor substrate for native L-rhamnose metabolism. This study aimed to identify such an inducer of $r h a B A D$ by screening 35 structural analogues of L-rhamnose. These candidates were screened, the genetic architecture of the rhaBAD expression system was simplified to improve its inducer compatibility, and the induction characteristics of the most promising candidates were studied in detail. Several rare sugars caused substantial induction of rhaBAD. L-Mannose in particular was found to be an excellent, metabolically orthogonal inducer of the rhaBAD expression system, superior to the natural inducer L-rhamnose.

\section{RESULTS}

Construction and Validation of a rhaBAD Reporter Construct. To evaluate the ability of candidate compounds to induce the $r h a B A D$ promoter, an assay system was required . To this end, we constructed a reporter plasmid (pCK301) containing a transcriptional fusion between the rhaBAD promoter of E. coli MG1655 and a gene encoding the highly stable and widely used "superfolder" variant of green fluorescent protein, sfGFP. ${ }^{47}$ In this arrangement, fluorescence depends upon transcription from the rhaBAD promoter, so the effects of candidate inducers can be readily determined. A similar plasmid lacking the reporter, pCK300, was constructed as a control. E. coli MG1655 was used as the host for induction assays, as it has been shown that the levels of RhaS and RhaR expressed from the native rhaSR operon in this strain are sufficient for full induction by the native inducer L-rhamnose, ${ }^{48}$ and because this fully sequenced strain contains no mutations in the rhaSR, rhaBAD, or rhaT operons relevant to this study. Before using this assay to evaluate untested candidate compounds, we first validated the reporter plasmid pCK301 using the native inducer L-rhamnose. This allows us to compare the response of the rhaBAD promoter of pCK301 to previously reported data. To demonstrate induction by L-rhamnose, and to assess the dependence of the response on L-rhamnose concentration, E. coli MG1655 cells containing pCK301 were cultured in complex medium (LB) supplemented with various concentrations of L-rhamnose for $16 \mathrm{~h}$, and then fluorescence (normalized to cell density) was determined as a measure of the sfGFP protein concentration (Figure 1). The fluorescence at the end of induction showed a highly linear relationship with Lrhamnose concentration (Figure $1 ; R^{2}$ value $=0.97$ ). Fluorescence in the absence of inducer was identical to the negative control plasmid pCK300 (no statistically significant difference; $P>0.05$ ), indicating very low basal expression. The linear concentration dependence and very low basal expression are both in excellent agreement with published data, ${ }^{16,23}$ which validates pCK301 as an appropriate system to test candidate inducer compounds.

Screening Analogues of L-Rhamnose as Inducers of the rhaBAD Promoter System. Thirty-five sugars structurally related to L-rhamnose (Figure 2) were investigated as potential inducers of the rhaBAD promoter system: (i) the four epimers (having one different chiral center from rhamnose) and three other diastereomers of rhamnose (changes of stereochemistry from rhamnose shown in red); (ii) a series of eight sugars with the same stereochemistry at $\mathrm{C} 2, \mathrm{C} 3$, and $\mathrm{C} 4$ as that of $\mathrm{L}-$ rhamnose (shown in purple); (iii) all eight isomeric pentoses and four pentitols (shown in green); and (iv) nine sugars in which the $\mathrm{C} 2 \mathrm{OH}$ group of L-rhamnose is replaced by other groups (shown in blue). Accordingly these cannot be isomerized by RhaA.

The series of 35 sugar mimics of L-rhamnose were screened for activity as inducers of the rhaBAD promoter, using the validated sfGFP reporter plasmid pCK301 and the end point fluorescence assay described above. To maximize sensitivity and 


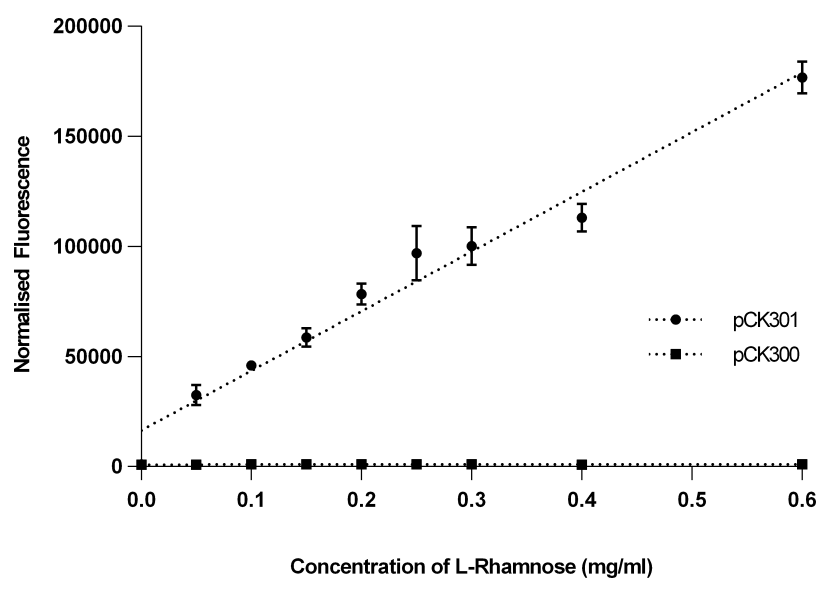

Figure 1. Validation of the $\mathrm{P}_{r h a B A D}$ reporter plasmid pCK301 by determining fluorescence response to L-rhamnose inducer concentration in complex media. E. coli MG1655 cells were transformed with either pCK301 ( $\mathrm{P}_{\text {rhaBAD }}-S f G F P$ transcriptional reporter $)$ or the control plasmid pCK300 (lacking $\mathrm{P}_{\text {rhaBAD }}$ and $s f G F P$ ), grown in $500 \mu \mathrm{L} \mathrm{LB}$ media cultures supplemented with different concentrations of Lrhamnose. Optical density at $600 \mathrm{~nm}$ and GFP fluorescence (excitation $488 \mathrm{~nm}$; emission $530 \mathrm{~nm}$ ) were measured after $16 \mathrm{~h}$. Lines shown represent a linear regression of each plot of normalized fluorescence $\left(\mathrm{GFP} / \mathrm{OD}_{600 \mathrm{~nm}}\right)$. A linear dose response to L-rhamnose is observed for pCK301 samples ( $R^{2}$ value: 0.97). Error bars shown are the standard deviation of three independent biological replicates.

allow detection of even weak induction, the compounds were used at a high concentration of $1 \mathrm{mg} / \mathrm{mL}$. Preliminary experiments revealed that most of the compounds were completely inactive as inducers of the rhaBAD promoter in this assay system. However, several compounds did induce expression, as shown in Figure 3, which also includes Lrhamnose for comparison. None of the analogues resulted in induction to the same level as L-rhamnose. However, four sugars showed potentially useful induction of the system after $16 \mathrm{~h}$ of growth in complex media: two epimers at C3 and C5 of rhamnose (6-deoxy-L-altrose and 6-deoxy-D-gulose respectively); and L-mannose and L-lyxose in which the $\mathrm{C} 6$ methyl group of rhamnose is replaced by $\mathrm{CH}_{2} \mathrm{OH}$ and $\mathrm{H}$, respectively.

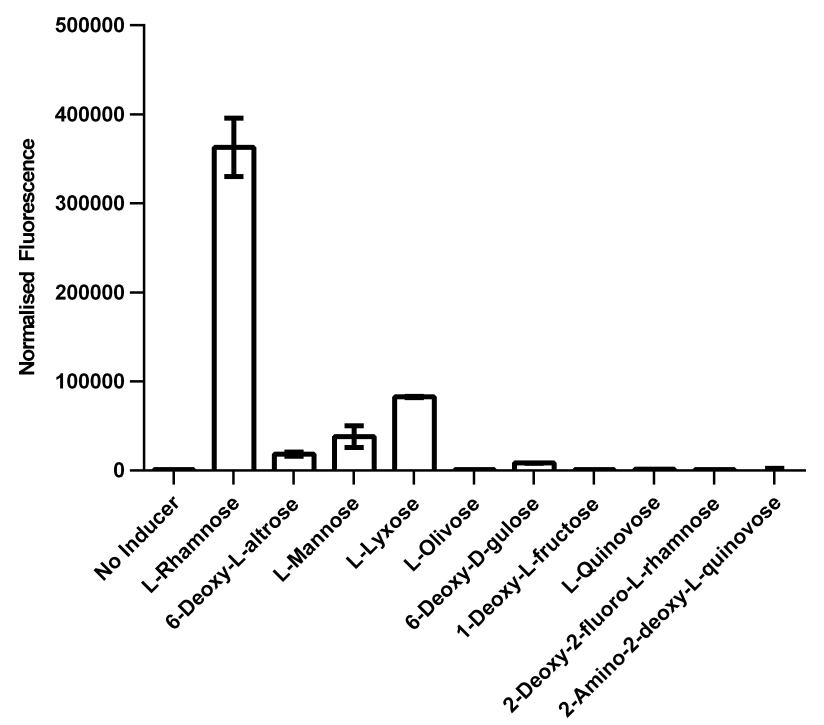

Figure 3. Initial screen of rhamnose analogues as inducers of the rhaBAD promoter system. Rhamnose analogues were screened for their ability to induce expression of the fluorescent reporter. E. coli MG1655 cells were transformed with pCK301, cultured for $16 \mathrm{~h}$ at 37 ${ }^{\circ} \mathrm{C}$ in LB media with each inducer at a final concentration of $1 \mathrm{mg} / \mathrm{mL}$. Optical density $(600 \mathrm{~nm})$ and GFP fluorescence was measured at the end of the experiment. Error bars shown are the standard deviation of three independent biological replicates. Statistical significance was determined using a one-way ANOVA, followed by a Tukey's multiple comparison test assuming unequal variance. Analogues which caused no induction (zero fluorescence values) are not shown, except those compared to results later in the study.

Decoupling the rhaBAD Promoter from the rhaSR Regulatory Cascade Reveals Effective Inducers. In the native regulatory cascade, L-rhamnose binds both to RhaR, inducing expression of the rhaSR operon, and to RhaS, inducing expression of the rhaBAD operon. To be an effective inducer of the native system, a compound must act via both $\mathrm{RhaR}$ and RhaS appropriately. These two proteins share only $36 \%$ identity and $54 \%$ similarity with each other, and Lrhamnose binding to the $\mathrm{N}$-terminal domains results in

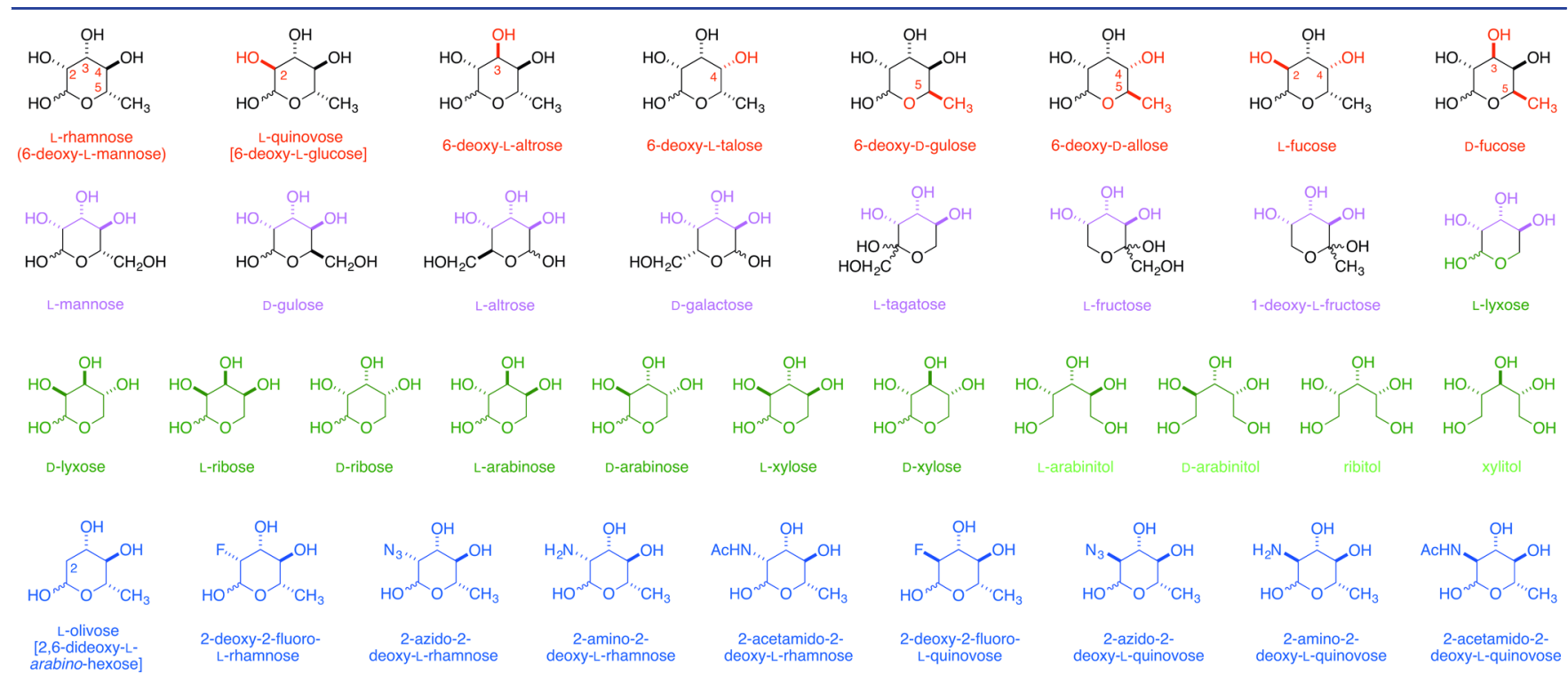

Figure 2. Structural analogues of L-rhamnose, as described in the text. 

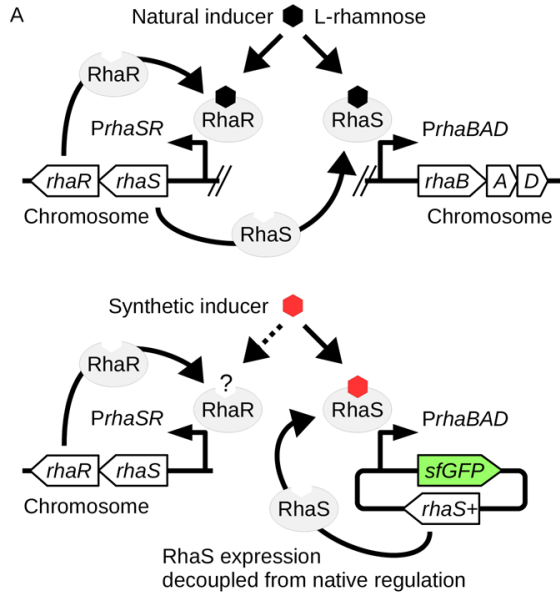

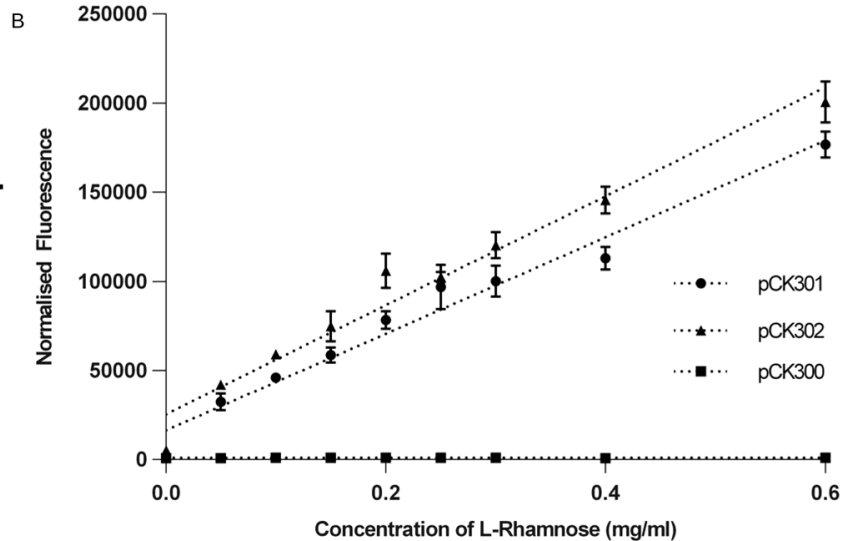

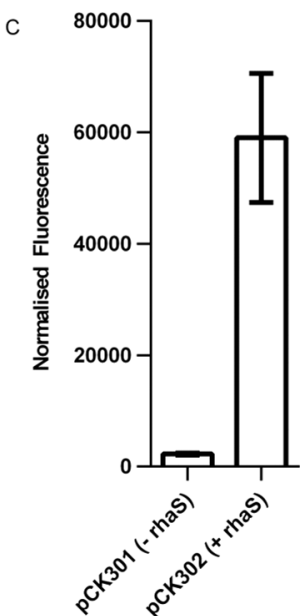

Figure 4. Design and evaluation of improved, rhaS-encoding reporter plasmid, pCK302. (a) Design of pCK302. It was hypothesized that weak induction of $\mathrm{P}_{r h a B A D}$ resulted from poor rhaS expression, which in turn was due to suboptimum interaction of candidate inducers with RhaR. A new reporter plasmid, pCK302, was thus constructed that expresses the MG1655 rhaS gene constitutively (rhaS+), insulating it from the native rhaSR regulatory cascade. (b) Induction response of the rhaBAD promoter to L-rhamnose concentration using the rhaS-expressing reporter plasmid pCK302. E. coli MG1655 cells were transformed with either pCK302 (+rhaS), pCK301 (-rhaS) or the control plasmid pCK300 (lacking $\mathrm{P}_{\text {rhaBAD }}$ and $s f G F P$ ), and transformants cultured for $16 \mathrm{~h}$ at $37^{\circ} \mathrm{C}$ in LB medium supplemented with different concentrations of L-rhamnose. Optical density (600 $\mathrm{nm}$ ) and GFP fluorescence were measured at the end of the experiment. Lines shown represent a linear regression of each plot of normalized fluorescence $\left(\mathrm{GFP} / \mathrm{OD}_{600 \mathrm{~nm}}\right)$. pCK302 samples demonstrate a linear dose-response to L-rhamnose with an $R^{2}$ value of 0.97 , identical to pCK301 samples. Error bars shown are the standard deviation of three independent biological replicates. (c) Relief of catabolite repression through the constitutive expression of rhaS on pCK302. E. coli MG1655 cells were transformed with either pCK301 (-rhaS) or pCK302 (+rhaS) and grown at $37{ }^{\circ} \mathrm{C}$ in M9 minimal media supplemented with $0.2 \%(\mathrm{w} / \mathrm{v})$ glucose and $0.4 \mathrm{mg} / \mathrm{mL}$ of L-rhamnose. Fluorescence and optical density and measurements were taken after $16 \mathrm{~h}$. Fluorescence normalized to $\left(\mathrm{GFP} / \mathrm{OD}_{600 \mathrm{~nm}}\right)$ is shown. The pCK302 samples were 25-fold more fluorescent than pCK301 samples when induced with L-rhamnose $(P<0.05$; statistical significance was determined using an unpaired $t$ test $)$. No fluorescence was observed in the absence of L-rhamnose. Error bars shown are the standard deviation of three independent biological replicates.

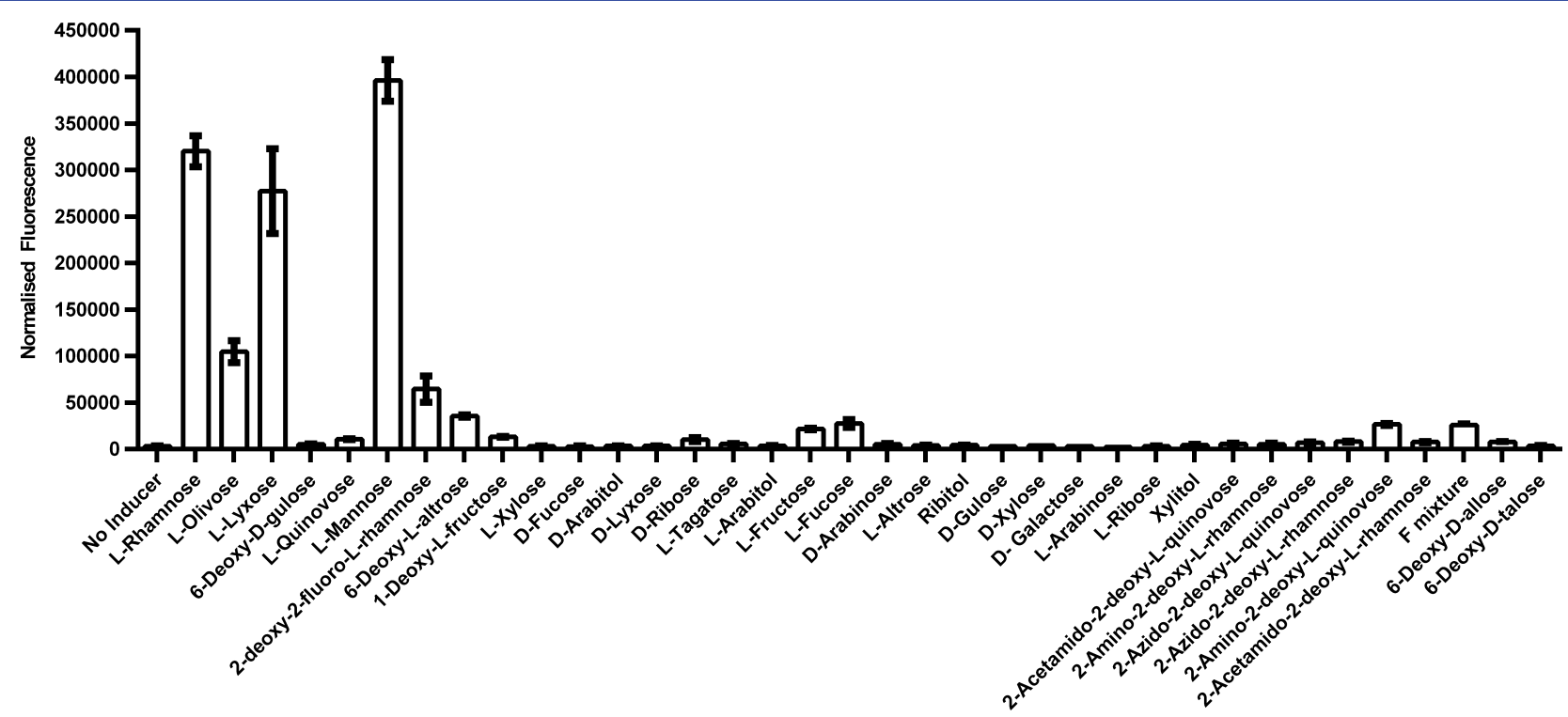

Figure 5. Screen of rhamnose analogues as inducers of the modified rhaBAD system expressing rhaS constitutively. All previously tested rhamnose analogues were screened again using the new rhaS-expressing reporter plasmid pCK302. E. coli MG1655 cells were transformed with pCK302 and transformants cultured for $16 \mathrm{~h}$ at $37^{\circ} \mathrm{C}$ in LB media supplemented with the analogue to be tested at a final concentration of $1 \mathrm{mg} / \mathrm{mL}$. Optical density $(600 \mathrm{~nm})$ and fluorescence were measured at the end of experiment. Error bars shown are the standard deviation of three independent biological replicates. Statistical significance was determined using a one-way ANOVA, followed by a Tukey's multiple comparison test assuming unequal variance. L-Rhamnose and five analogues showed statistically significant induction using this improved reporter plasmid.

conformational changes that affect different helix-turn-helix motifs (HTH) in the DNA-binding, C-terminal domains of each protein. ${ }^{49}$ For these reasons, it is quite feasible that a compound might properly bind and induce the correct conformational change in RhaS, but not in RhaR, or vice versa. Such compounds would be expected to give weak or negative results in our initial screen described above, which depended upon the native rhaSR regulatory cascade. However, it would be useful to identify compounds that bind well to RhaS, even if they bind poorly to RhaR, as these could be effective inducers of the rhaBAD promoter in synthetic systems that do not depend upon the native regulatory cascade for 
production of RhaS (Figure 4A). ${ }^{50}$ To investigate this, we implemented such a synthetic system by constructing a modified reporter plasmid pCK302 (Figure 4A) containing a constitutively expressed copy of the rhaS gene (referred to herein as rhaS+). This effectively decouples induction of the rhaBAD promoter from dependence on induction of rhaSR by $\mathrm{RhaR}^{34}$

The new decoupled reporter plasmid pCK302 (including rhaS+) was validated using the end-point fluorescence induction assay with various concentrations of L-rhamnose, as before (Figure 4B). E. coli MG1655 cells containing pCK302 performed very similarly to those containing pCK301, showing the desired linear relationship between L-rhamnose concentration and fluorescence of sfGFP $\left(R^{2}=0.97\right)$ and a barely detectable basal level of fluorescence in the absence of inducer (Figure 4B). This confirmed that the reporter system remained a suitable assay following the addition of rhaS+, so the decoupled reporter plasmid pCK302 was used for all subsequent reporter experiments. Interestingly, expression of rhaS+ from pCK302 also substantially relieved catabolite repression of the rhaBAD reporter. E. coli MG1655 cells containing pCK302 (rhaS+) cultured overnight in LB medium supplemented with both $0.4 \mathrm{mg} / \mathrm{mL}$ L-rhamnose and $0.2 \% \mathrm{D}$ glucose showed 25-fold higher fluorescence than equivalent cultures of cells containing pCK301, which lacks rhaS (Figure $4 \mathrm{C}$, fluorescence statistically significantly different, $P<0.05$ ). This will allow the rhaBAD promoter to be used in combination with glucose, which will be useful particularly in situations in which use of noncatabolite-repressing substrates such as glycerol is complicated or unsuitable, such as in E. coli anaerobic fermentation, which is an important metabolic mode for chemical production.

Using the decoupled reporter plasmid pCK302, all 35 analogues of L-rhamnose were screened again for activity as inducers of the rhaBAD promoter as before. In this second screen (Figure 5), we observed induction of sfGFP expression by several additional compounds that did not induce expression in the previous screen using pCK301 (Figure 3), including the non-natural sugars L-olivose and 2-deoxy-2-fluoro-L-rhamnose. All of the compounds identified as modest inducers in the previous screen (Figure 3) showed greater induction in this second screen (Figure 5). Among these, L-lyxose induced fluorescence to a level almost equal to that of L-rhamnose, whereas L-mannose actually induced fluorescence to a significantly greater level than the natural inducer L-rhamnose $(P<0.05)$. This suggested that, at least in this assay system, either induction of the rhaBAD promoter is more sensitive to $\mathrm{L}$ mannose than the natural inducer L-rhamnose, or that there are differences in the kinetics of induction by these two compounds, or both.

Characterization and Comparison of Promising Inducers. We chose the five strongest analogue inducers from the second end-point fluorescence screen (Figure 5) for further characterization as candidate orthogonal inducers. These promising compounds were L-mannose, L-lyxose, Lolivose, 2-deoxy-2-fluoro-L-rhamnose and 6-deoxy-L-altrose. First, the concentration dependence of induction by each of these analogues was determined using the same range of concentrations tested previously for L-rhamnose (Figure 6A). An additional, lower range of concentrations was also included for L-mannose to determine the concentration dependence of this analogue at the lower fluorescence levels reached with the other analogues (Figure 6A, inset). All analogues showed a
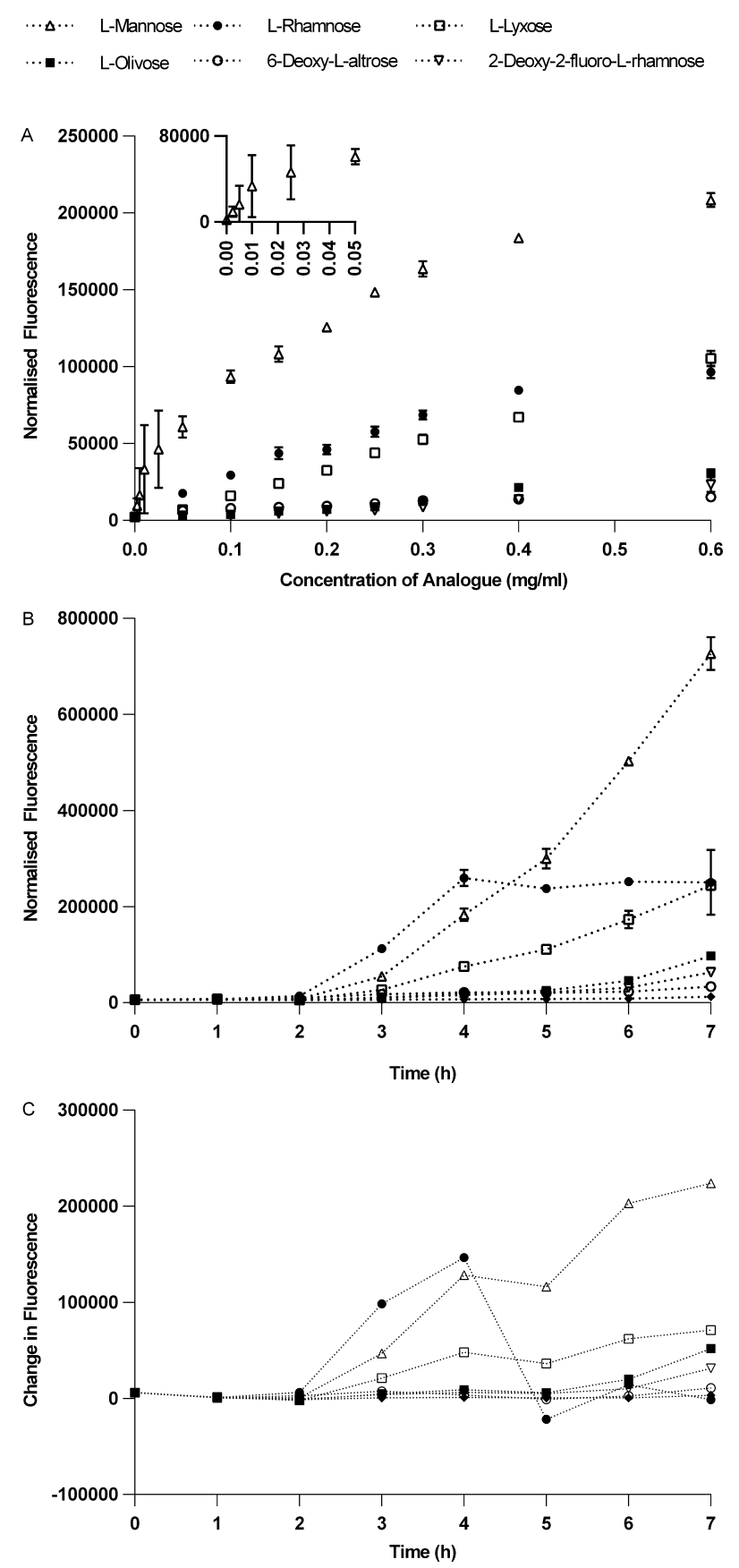

Figure 6. Characterization of the most promising analogue inducers: response to inducer concentration and induction kinetics. (a) Response of the $r h a B A D$ promoter to analogue inducer concentrations. The five analogues showing statistically significant induction (L-mannose, L-lyxose, L-olivose, 6-deoxy-L-altrose, and 2-deoxy-2fluoro-L-rhamnose) were added to $500 \mu \mathrm{L}$ cultures of MG1655 containing pCK302 to final concentrations of $0.05-0.6 \mathrm{mg} / \mathrm{mL}$ and optical density and fluorescence measured after $16 \mathrm{~h}$ at $37{ }^{\circ} \mathrm{C}$. The dose response of L-mannose was further characterized at lower concentrations (inset). Error bars shown are standard deviation of three independent biological replicates. (b) Kinetics of rhaBAD promoter induction by novel analogue inducers. E. coli MG1655 cells transformed with pCK302 were grown in $500 \mu \mathrm{L}$ of LB media cultures supplemented with $0.4 \mathrm{mg} / \mathrm{mL}$ of the five analogue inducers for $7 \mathrm{~h}$ at $37^{\circ} \mathrm{C}$ and optical density and fluorescence measurements taken every hour. Error bars shown are the standard deviation of three independent biological replicates. (c) Change in mean fluorescence over time as a measure of the rate of sfGFP synthesis. 
similar linear response to concentration as L-rhamnose (Figure $6 \mathrm{~A}, R^{2}$ values of linear regression: 0.86-0.99).

Next, the kinetics of induction of each of the five promising analogues was studied by monitoring the fluorescence of cultures (of E. coli MG1655 cells containing pCK302 in LB medium, as before) containing $0.4 \mathrm{mg} / \mathrm{mL}$ of inducer over the course of $7 \mathrm{~h}$ (Figure 6B). Use of sfGFP in this study allows additional interpretation of the kinetic data, as sfGFP is very stable, ${ }^{47}$ so it is a reasonable approximation to treat degradation of the sfGFP protein during the course of this experiment as negligible. Therefore, fluorescence at a given time is a measure of the cumulative total of sfGFP protein produced by that time (Figure 6B), and hence the change in fluorescence per unit time (that is, the first derivative of fluorescence with respect to time) is a measure of the rate of sfGFP production (Figure 6C) due to transcription from the rhaBAD promoter of pCK302. The kinetics of induction of the $r h a B A D$ promoter by the native inducer L-rhamnose differed qualitatively in two ways from the kinetics of induction of all the other inducers (Figure $6 C)$. First, induction by L-rhamnose occurred slightly earlier than the other analogues, being detectable after $2 \mathrm{~h}(P<0.05)$, and already approaching its maximum rate by $3 \mathrm{~h}$. Second and more importantly, induction by L-rhamnose was transient, giving no further increase in fluorescence after $4 \mathrm{~h}$, indicating that transcription from the $r h a B A D$ promoter had stopped. In marked contrast, induction by the five promising analogues (especially L-mannose and L-lyxose) was sustained to the end of the experiment. Among the quantitative differences, the most noteworthy is that the relative rate of sfGFP production (increase of fluorescence; Figure 6C) for L-mannose at its maximum (at $7 \mathrm{~h}$ ) was greater than the rate for the natural inducer L-rhamnose at its maximum (at $4 \mathrm{~h}$ ). Hence this experiment indicates that differences between L-rhamnose and L-mannose in both the strength and the kinetics of induction contribute to the different end-point fluorescence assay results noted previously (Figure 5).

Sustained induction kinetics by a given inducer are likely to be explained by poor or absent metabolism of that compound. To test if the five promising inducers of the rhaBAD promoter were efficiently metabolized by $E$. coli MG1655 containing the reporter plasmid pCK302, growth assays at $37^{\circ} \mathrm{C}$ were carried out using M9 minimal medium agar plates, each supplemented with $0.4 \%(\mathrm{w} / \mathrm{v})$ of one of the analogues as the sole carbon source. Plates supplemented with glycerol and plates lacking a carbon source were used as positive and negative controls, respectively. Cells grew fastest, forming the largest colonies, on the plates containing glycerol. Both L-rhamnose and L-lyxose supported good growth of E. coli. Growth on the other analogues including L-mannose was much slower, with very small colonies observed after incubation at $37^{\circ} \mathrm{C}$ for $48 \mathrm{~h}$ (data not shown). These observations suggest that the most promising strong inducer L-mannose is poorly metabolized. To confirm this, growth assays at $37{ }^{\circ} \mathrm{C}$ were carried out using M9 minimal medium liquid cultures, each supplemented with $0.4 \%(\mathrm{w} / \mathrm{v})$ L-mannose, L-rhamnose, or glycerol as sole carbon source (Figure 7). L-Mannose did not support growth of E. coli, whereas L-rhamnose or glycerol did support growth.

Finally, flow cytometry was used to assess whether the most promising analogue, L-mannose, and the natural inducer, Lrhamnose, led to unimodal or multimodal induction of the rhaBAD promoter. Induction that leads to subpopulations of cells with different distributions of expression levels is said to be multimodal. Unimodality is typically preferred, as it means that

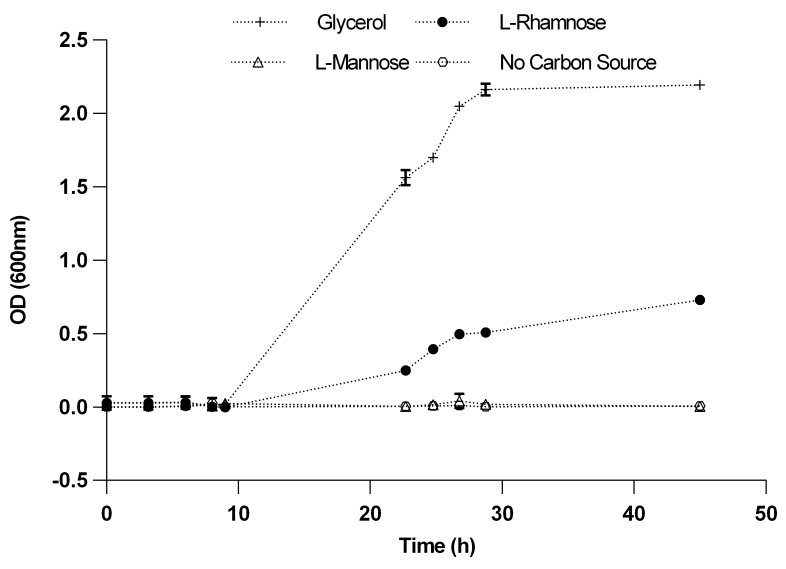

Figure 7. Assessment of growth of E. coli with selected inducers as sole carbon source. E. coli MG1655 cells transformed with pCK300 were cultured at $37^{\circ} \mathrm{C}$ for $45 \mathrm{~h}$ in $\mathrm{M} 9$ minimal media supplemented with one of the following sole carbon sources: $0.4 \%(\mathrm{w} / \mathrm{v})$ glycerol, $0.4 \%$ $(\mathrm{w} / \mathrm{v})$ L-rhamnose, $0.4 \%(\mathrm{w} / \mathrm{v})$ L-mannose, no carbon source. Samples were taken at various time points, and the optical density at $600 \mathrm{~nm}$ was recorded. Error bars shown are the standard deviation of three independent biological replicates.

an inducer can be used to achieve a similar concentration of protein(s) of interest in all cells in a population, rather than different concentrations. E. coli MG1655 cells containing pCK302 were grown to the mid $(6 \mathrm{~h})$ and late $(8 \mathrm{~h})$ exponential growth phase in LB medium supplemented with three concentrations of L-rhamnose or L-mannose $(0.2,0.4$, or $0.6 \mathrm{mg} / \mathrm{mL})$, and the fluorescence of cells in samples were measured by flow cytometry (Figure 8). The number of fluorescing and nonfluorescing cells in each sample were counted at each time point for three independent biological replicates. As expected, L-rhamnose induction led to a unimodal distribution of expression for all three inducer concentrations tested and at both time points. Induction with all three concentrations of L-mannose led to a unimodal distribution of expression at the $8 \mathrm{~h}$ time point. At the $6 \mathrm{~h}$ time point, Lmannose induction led to a unimodal distribution at the medium $(0.4 \mathrm{mg} / \mathrm{mL})$ and high $(0.6 \mathrm{mg} / \mathrm{mL})$ concentrations, but biomodality was observed at the low $(0.2 \mathrm{mg} / \mathrm{mL})$ concentration.

\section{DISCUSSION}

This study sought and identified several inducers of the $r h a B A D$ promoter of $E$. coli that enable sustained induction from this promoter for much longer durations than the native inducer L-rhamnose. To varying degrees, these compounds can be considered functionally orthogonal to E. coli metabolism, as they are evidently not metabolized well enough to cause transient induction, unlike the native inducer L-rhamnose. Overall, L-mannose seems likely to be the most useful of the compounds studied, as it provides the greatest range of expression levels, while retaining very good linearity of response to inducer concentration, and sustained induction. L-Lyxose may also prove useful, particularly at lower expression levels, where it may offer slightly greater control than L-mannose (Figure 6). L-Mannose and L-lyxose are commercially available, unlike most of the other compounds tested, which is an important advantage for their usefulness to most researchers. The only observed disadvantage of L-mannose was bimodality at the lowest inducer concentration tested, at the early time 

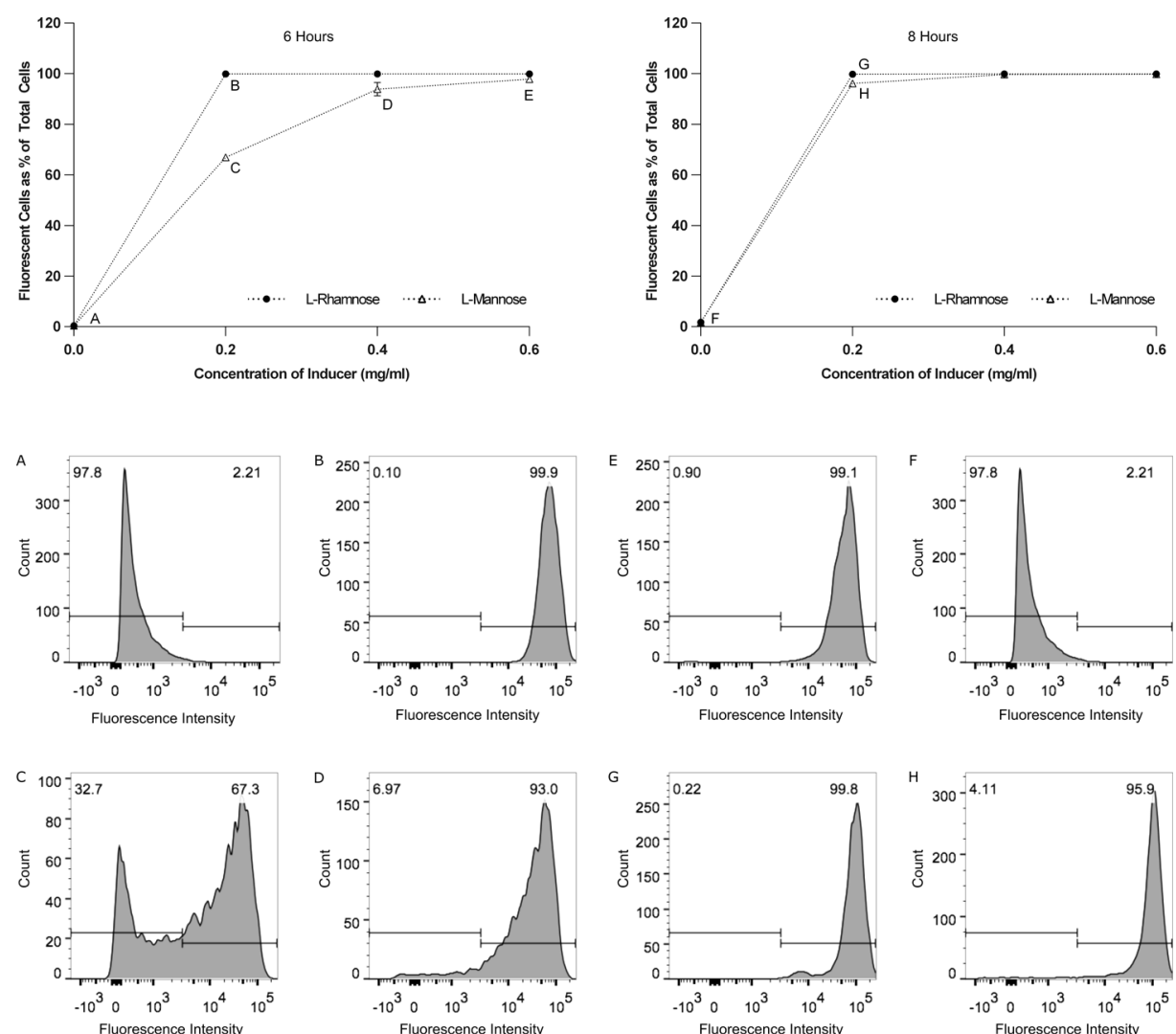

Figure 8. Distribution of expression levels in populations of cells induced by L-rhamnose or L-mannose. E. coli MG1655 cells transformed with pCK302 were grown in $300 \mu \mathrm{L}$ cultures of LB media supplemented with $0.2,0.4$, or $0.6 \mathrm{mg} / \mathrm{mL}$ of L-rhamnose or L-mannose at $37^{\circ} \mathrm{C}$. After 6 and 8 $\mathrm{h}$, representing the mid to late exponential phase of growth, cells were analyzed using a LSRFortessa flow cytometer (BD Biosciences). Cells were gated appropriately, and GFP fluorescence was measured at each time point and inducer concentration. Histograms of fluorescence intensity were plotted, and population statistics were extracted. The percentages of events within the fluorescent and nonfluorescent populations are indicated on each histogram. Error bars shown are the standard deviation of three independent biological replicates.

point $(6 \mathrm{~h})$. This bimodality may be an issue for some uses. It is known that bimodality occurs in induction of the lac promoter by $\mathrm{IPTG}^{51,52}$ and induction of the $\operatorname{araBAD}$ promoter by arabinose $^{53}$ due to positive feedback between uptake of each inducer and inducible expression of the corresponding uptake system. L-Rhamnose catabolism involves the inducible expression of the uptake system RhaT, so this might explain how rhaBAD induction could develop bimodality by this mechanism.

The panel of 35 analogues of L-rhamnose studied here induced expression of the rhaBAD promoter to a wide range of levels. It was surprising to observe the apparent greater sensitivity of the rhaBAD reporter system pCK302 to Lmannose than to the natural inducer L-rhamnose. As crystal structures of RhaS and RhaR are not currently available, it is difficult to provide meaningful structural interpretation of these observations. However, it is clear that induction of this system, governed by ligand binding to $\mathrm{RhaS}$ and/or RhaR, is very sensitive to small changes in the structure of the ligand, including its stereochemistry. If crystal structures of RhaS or RhaR are obtained in future, it would be interesting to reanalyse these data. Differences in rates of uptake of these compounds by $\mathrm{RhaT}^{37}$ and other transporters might also contribute to differences in the induction observed.

It is interesting that decoupling expression of rhaS from dependence on RhaR and the rhaSR promoter, effectively bypassing the native cascade, did not substantially change the shape, maximum or minimum of the induction response to inducer concentration (Figure 4B). This suggests that the native architecture of the rhaSR cascade is not required for these properties, so presumably exists chiefly for reasons of resource efficiency, as in the uninduced state it minimizes protein expression from rhaSR as well as rhaBAD.

The results of this study place the rhaBAD expression system in the small group of preferred inducible systems for which effective orthogonal inducers are available. The additional control afforded by sustained induction represents a substantial improvement in the usefulness of the rhaBAD system for synthetic biology. Due to this, and the other highly desirable induction properties of the $r h a B A D$ system, it may now be the best choice of inducible expression system in many cases. It has been shown that the rhaBAD system can be used beyond its native host by providing RhaS in trans, so the inducers reported here are likely to be useful, and orthogonal to metabolism, in organisms beyond E. coli.

This study highlights the synergy between synthetic biology and synthetic chemistry. The combined approach used here could readily be applied to other expression systems to identify useful combinations of orthogonal inducers and compatible genetic configurations. Although this report has focused on identification of the single most effective orthogonal inducer by typical criteria, the same approach could be used to identify a set of inducers with different induction characteristics. LRhamnose and L-mannose represent a minimal example of such a set, as L-rhamnose causes transient induction whereas Lmannose causes sustained induction. Such sets of inducers 
could be used to interrogate or optimize genetic circuits or networks instead of, or in parallel to, genetic modifications and redesigns. For example, the inducers reported here may have proven useful in optimization of a recently described synthetic system using the $r h a B A D$ promoter and RhaS, which required a series of design iterations to achieve the desired functionality. ${ }^{50}$ The use of alternative chemical inducers with differing induction characteristics represents an under-utilized and rarely considered $^{54}$ mechanism for predictable external control of gene expression, which could be better developed and exploited as demonstrated here.

\section{MATERIALS AND METHODS}

Rare Sugars. The sugars were prepared either from rhamnose ${ }^{55}$ or by isomerization of other hexoses using enzymatic methods. ${ }^{56-58}$

Bacterial Strains and Growth Conditions. E. coli strain DH5 $\alpha$ was used for all plasmid construction and propagation. Wild-type E. coli strain MG1655 was used for all induction assays. Cells were cultured in LB or M9 minimal media at 37 ${ }^{\circ} \mathrm{C}$. Ampicillin was used at a final concentration of $125 \mu \mathrm{g} / \mathrm{mL}$ where appropriate.

Plasmid Construction. A list of plasmids and oligonucleotides is provided in Table S1. Sequences of pCK300, pCK301, and pCK302 have been submitted to Genbank, accession numbers are KU555408, KU555409, and KU555410, respectively. The Gibson assembly method was used throughout. ${ }^{59}$ To construct the initial reporter plasmid pCK301, the E. coli rhaBAD promoter $\left(\mathrm{P}_{\text {rhaBAD }}\right)$ was amplified from E. coli MG1655 genomic DNA using oligonucleotides oligoCK207 and oligoCK208 and inserted in place of the T5 promoter on the medium copy number E. coli expression vector pJ404 (DNA2.0), which itself was amplified using oligonucleotides oligoCK97 and oligoCK98. Next, superfolder GFP ( $s f G F P$ ) was amplified from pJT118-sfGFP ${ }^{60}$ using oligonucleotides oligoCK209 and oligoCK210 and inserted immediately downstream of this promoter, following amplification of the vector using oligoCK56 and oligoCK207. An appropriate ribosomebinding site sequence with a translation initiation rate (TIR) of 50000 was designed using the ribosome binding site calculator, $^{61}$ and was incorporated using the forward primer (oligoCK210). pCK302 was constructed through amplification of the native rhaS gene of E. coli including its native ribosomebinding sequence using oligonucleotides oligoCK173 and oligoCK174, which was inserted immediately downstream of $a m p R$ (vector amplified using oligonucleotides oligoCK171 and oligoCK172) to ensure constitutive expression from the $a m p R$ promoter. pCK300 was constructed by removal of the $\mathrm{P}_{\text {rhaBAD- }}$ sf GFP fragment of pCK301 using oligonucleotides oligoCK170 and oligoCK91. All plasmids were verified by nucleotide sequencing.

Assays. To test for induction of the rhaBAD promoter by the analogues, MG1655 was transformed with either pCK300, pCK301, or pCK302. Successful transformants were isolated and cultured in $5 \mathrm{~mL}$ of LB and ampicillin with shaking at 37 ${ }^{\circ} \mathrm{C}$ for $16 \mathrm{~h}$. These precultures were used to inoculate all subsequent assay cultures throughout this work. A Synergy HT platereader was used for all absorbance and fluorescence measurements. Optical density (OD) was measured at A600 $\mathrm{nm}$, and fluorescence excitation and emission wavelengths used were 488 and $530 \mathrm{~nm}$, respectively.

End-point induction assays were performed in $500 \mu \mathrm{L} \mathrm{LB}$ cultures supplemented with ampicillin and various concen- trations of L-rhamnose or analogues and grown at $37{ }^{\circ} \mathrm{C}$ in deep 96-well plates with shaking for $16 \mathrm{~h}$. Cultures were then centrifuged at $4009 \mathrm{~g}$ for $10 \mathrm{~min}$ and resuspended in phosphate buffered saline and diluted 1 in $4 ; 200 \mu \mathrm{L}$ was used to measure optical density and fluorescence. Fluorescence readings were normalized by OD.

Time course experiments of $\mathrm{P}_{\text {rhaBAD }}$ induction in LB were performed as follows: $300 \mu \mathrm{L}$ of $\mathrm{LB}$ media supplemented with ampicillin and $0.4 \mathrm{mg} / \mathrm{mL}$ of inducer, was inoculated with $1 /$ 1000 volume of the preculture and grown at $37{ }^{\circ} \mathrm{C}$ in 96 -well microplates with shaking for $7 \mathrm{~h}$. Optical density and fluorescence readings were taken every $60 \mathrm{~min}$. Fluorescence readings were normalized to OD.

Catabolite repression was assayed by transforming E. coli MG1655 cells with either pCK301 or pCK302, and transformants were used to inoculate $500 \mu \mathrm{L}$ of liquid M9 minimal media cultures supplemented with $0.2 \%$ glucose and $0.4 \%$ of Lrhamnose as inducer and grown at $37^{\circ} \mathrm{C}$ in deep 96-well plates with shaking for $16 \mathrm{~h} ; 200 \mu \mathrm{L}$ of the culture was used to measure optical density and fluorescence.

To test whether the analogues were capable of supporting growth of E. coli cells as the sole carbon source, MG1655 cells were transformed with pCK302 and transformants streaked out on M9 minimal media agar plates supplemented with $0.2 \%(\mathrm{w} /$ $\mathrm{v}$ unless otherwise stated) final concentration of a single carbon source: glycerol (v/v), L-rhamnose, L-lyxose, L-mannose, 6deoxy-L-altrose, L-olivose, 2-deoxy-2-fluoro-L-rhamnose or no sugar. Plates were incubated at $37{ }^{\circ} \mathrm{C}$ for $72 \mathrm{~h}$ at which time growth was scored qualitatively. Additionally $3.3 \mathrm{~mL}$ of liquid M9 minimal media cultures with either $0.2 \%$ glycerol (v/v), Lrhamnose $(\mathrm{w} / \mathrm{v})$. or L-mannose $(\mathrm{v} / \mathrm{v})$ were incubated for 4 days at $37^{\circ} \mathrm{C}$, and $100 \mu \mathrm{L}$ samples were taken at regular time points for optical density measurements.

To test whether L-rhamnose or L-mannose induction led to a bimodal expression profile across a population, MG1655 cells were transformed with $\mathrm{pCK} 302$, and transformants were cultured in $300 \mu \mathrm{L}$ of LB supplemented with ampicillin and various concentrations of L-rhamnose or L-mannose, at $37^{\circ} \mathrm{C}$ in deep 96-well plates with shaking. At the 6 and $8 \mathrm{~h}$ time points, cells were diluted 1 in 100 in phosphate-buffered saline and analyzed using a LSRFortessa flow cytometer (BD Biosciences). Cells were gated using forward and side scatter, and GFP fluorescence (excitation and emission wavelengths: 488 and $525 \mathrm{~nm}$ [with $20 \mathrm{~nm}$ bandwidth] respectively) was measured at each time point and inducer concentration. Histograms of fluorescence intensity were plotted, and population statistics were extracted.

\section{ASSOCIATED CONTENT}

\section{Supporting Information}

The Supporting Information is available free of charge on the ACS Publications website at DOI: 10.1021/acssynbio.6b00030.

List of plasmids and oligonucleotides (PDF)

\section{AUTHOR INFORMATION}

\section{Corresponding Authors}

*E-mail: jheap@imperial.ac.uk.

*E-mail: yoshihara@ag.kagawa-u.ac.jp.

\section{Notes}

The authors declare no competing financial interest. 


\section{ACKNOWLEDGMENTS}

This work was supported by the Biotechnology and Biological Sciences Research Council [BB/M011321/1 to J.T.H.]; Galchimia S.A. [Project IN852A 2014/1 to R.J.E.]; and an Emeritus Leverhulme Research Fellowship to G.W.J.F. The authors thank George Taylor for advice on data analysis of flow cytometry data.

\section{REFERENCES}

(1) Guzman, L. M., Belin, D., Carson, M. J., and Beckwith, J. (1995) Tight regulation, modulation, and high-level expression by vectors containing the arabinose $\mathrm{P}(\mathrm{BAD})$ promoter. J. Bacteriol. 177, 41214130.

(2) Gronenborn, B. (1976) Overproduction of phage lambda repressor under control of the lac promotor of Escherichia coli. Mol. Gen. Genet. 148, 243-250.

(3) Brosius, J., Erfle, M., and Storella, J. (1985) Spacing of the -10 and -35 regions in the tac promoter. Effect on its in vivo activity. J. Biol. Chem. 260, 3539-3541.

(4) Skerra, A. (1994) Use of the tetracycline promoter for the tightly regulated production of a murine antibody fragment in Escherichia coli. Gene 151, 131-135.

(5) Blasi, B., Peca, L., Vass, I., and Kós, P. B. (2012) Characterization of stress responses of heavy metal and metalloid inducible promoters in synechocystis PCC6803. J. Microbiol. Biotechnol. 22, 166-169.

(6) Mueller, P. R., Salser, S. J., and Wold, B. (1988) Constitutive and metal-inducible protein:DNA interactions at the mouse metallothionein I promoter examined by in vivo and in vitro footprinting. Genes Dev. 2, 412-427.

(7) Egland, K. A., and Greenberg, E. P. (1999) Quorum sensing in Vibrio fischeri: elements of the luxI promoter. Mol. Microbiol. 31, 1197-1204.

(8) Fuqua, W. C., Winans, S. C., and Greenberg, E. P. (1994) Quorum sensing in bacteria: the LuxR-LuxI family of cell densityresponsive transcriptional regulators. J. Bacteriol. 176, 269-275.

(9) Schuster, M., Urbanowski, M. L., and Greenberg, E. P. (2004) Promoter specificity in Pseudomonas aeruginosa quorum sensing revealed by DNA binding of purified LasR. Proc. Natl. Acad. Sci. U. S. A. 101, 15833-15839.

(10) Elvin, C. M., Thompson, P. R., Argall, M. E., Hendry, P., Stamford, N. P., Lilley, P. E., and Dixon, N. E. (1990) Modified bacteriophage lambda promoter vectors for overproduction of proteins in Escherichia coli. Gene 87, 123-126.

(11) Khalameyzer, V., Fischer, I., and Bornscheuer, U. T. (1999) Biochemical Characterization of an Esterase from Pseudomonas fluorescens with High Activity towards Lactones Screening. Nucleotide Sequence, and Biochemical Characterization of an Esterase from Pseudomonas fluorescens with High Activity towards Lactones 65, 477482.

(12) Wilms, B., Wiese, A., Syldatk, C., Mattes, R., Altenbuchner, J., and Pietzsch, M. (1999) Cloning, nucleotide sequence and expression of a new L-N-carbamoylase gene from Arthrobacter aurescens 3747 in E. coli. J. Biotechnol. 68, 101-113.

(13) Wilms, B., Hauck, A., Reuss, M., Syldatk, C., Mattes, R., Siemann, M., and Altenbuchner, J. (2001) High-cell-density fermentation for production of L-N-carbamoylase using an expression system based on the Escherichia coli rhaBAD promoter. Biotechnol. Bioeng. 73, 95-103.

(14) Cardona, S. T., and Valvano, M. a. (2005) An expression vector containing a rhamnose-inducible promoter provides tightly regulated gene expression in Burkholderia cenocepacia. Plasmid 54, 219-228.

(15) Cardona, S. T., Mueller, C. L., and Valvano, M. A. (2006) Identification of essential operons with a rhamnose-inducible promoter in Burkholderia cenocepacia. Appl. Environ. Microbiol. 72, $2547-2555$

(16) Giacalone, M., Gentile, A., Lovitt, B., Berkley, N., Gunderson, C., and Surber, M. (2006) Toxic protein expression in Escherichia coli using a rhamnose-based tightly regulated and tunable promoter system. BioTechniques 40, 355-364.

(17) Haldimann, A., Daniels, L. L., and Wanner, B. L. (1998) Use of New Methods for Construction of Tightly Regulated Arabinose and Rhamnose Promoter Fusions in Studies of the Escherichia coli Phosphate Regulon. J. Bacteriol. 180, 1277-1286.

(18) Jeske, M., and Altenbuchner, J. (2010) The Escherichia coli rhamnose promoter $\mathrm{rhaP}(\mathrm{BAD})$ is in Pseudomonas putida KT2440 independent of Crp-cAMP activation. Appl. Microbiol. Biotechnol. 85, 1923-1933.

(19) Juhas, M., Eberl, L., and Church, G. M. (2012) Essential genes as antimicrobial targets and cornerstones of synthetic biology. Trends Biotechnol. 30, 601-607.

(20) Tamayo-Ramos, J. a., Flipphi, M., Pardo, E., Manzanares, P., and Orejas, M. (2012) L-rhamnose induction of Aspergillus nidulans $\alpha$-Lrhamnosidase genes is glucose repressed via a CreA-independent mechanism acting at the level of inducer uptake. Microb. Cell Fact. 11, 26.

(21) Englesberg, E., Irr, J., Power, J., and Lee, N. (1965) Positive control of enzyme synthesis by gene $\mathrm{C}$ in the $\mathrm{L}$-arabinose system. $J$. Bacteriol. 90, 946-957.

(22) Lee, N., and Englesberg, E. (1962) DUAL EFFECTS OF STRUCTURAL GENES IN ESCHERICHIA COLI. Proc. Natl. Acad. Sci. U. S. A. 48, 335.

(23) Terpe, K. (2006) Overview of bacterial expression systems for heterologous protein production: from molecular and biochemical fundamentals to commercial systems. Appl. Microbiol. Biotechnol. 72, 211-222.

(24) Tobin, J. F., and Schleif, R. F. (1987) Positive regulation of the Escherichia coli L-rhamnose operon is mediated by the products of tandemly repeated regulatory genes. J. Mol. Biol. 196, 789.

(25) Figge, J., Wright, C., Collins, C. J., Roberts, T. M., and Livingston, D. M. (1988) Stringent regulation of stably integrated chloramphenicol acetyl transferase genes by E. coli lac repressor in monkey cells. Cell 52, 713-722.

(26) Dvorak, P., Chrast, L., Nikel, P. I., Fedr, R., Soucek, K. Sedlackova, M., Chaloupkova, R., de Lorenzo, V., Prokop, Z., and Damborsky, J. (2015) Exacerbation of substrate toxicity by IPTG in Escherichia coli BL21(DE3) carrying a synthetic metabolic pathway. Microb. Cell Fact. 14, 201.

(27) Kosinski, M. J., Rinas, U., and Bailey, J. E. (1992) Isopropyl-B-dthiogalactopyranoside influences the metabolism of Escherichia coli. Appl. Microbiol. Biotechnol. 36, 782-784.

(28) Englesberg, E., Anderson, R. L., Weinberg, R., Lee, N., Hoffee, P., Huttenhauer, G., and Boyer, H. (1962) L-Arabinose-sensitive, Lribulose 5-phosphate 4-epimerase-deficient mutants of Escherichia coli. J. Bacteriol. 84, 137-146.

(29) Power, J. (1967) The L-rhamnose genetic system in Escherichia coli K-12. Genetics 55, 557-568.

(30) Moralejo, P., Egan, S. M., Hidalgo, E., and Aguilar, J. (1993) Sequencing and characterization of a gene cluster encoding the enzymes for L-rhamnose metabolism in Escherichia coli. J. Bacteriol. $175,5585-5594$.

(31) Egan, S. M., and Schleif, R. F. (1993) A Regulatory Cascade in the Induction of rhaBAD. J. Mol. Biol. 234, 87-98.

(32) Tobin, J. F., and Schleif, R. F. (1990) Transcription from the rha operon psr promoter. J. Mol. Biol. 211, 1-4.

(33) Vía, P., Badía, J., Baldomà, L., Obradors, N., and Aguilar, J. (1996) Transcriptional regulation of the Escherichia coli rhaT gene. Microbiology 142 (7), 1833-1840.

(34) Egan, S. M., and Schleif, R. F. (1993) A Regulatory Cascade in the Induction of rhaBAD. J. Mol. Biol. 234, 87-98.

(35) Wickstrum, J. R., Skredenske, J. M., Balasubramaniam, V., Jones, K., and Egan, S. M. (2010) The AraC/XylS family activator RhaS negatively autoregulates rhaSR expression by preventing cyclic AMP receptor protein activation. J. Bacteriol. 192, 225-232.

(36) Boronat, A., and Aguilar, J. (1979) Rhamnose-induced propanediol oxidoreductase in Escherichia coli: purification, proper- 
ties, and comparison with the fucose-induced enzyme. J. Bacteriol. 140, 320-326.

(37) Muiry, J. a., Gunn, T. C., McDonald, T. P., Bradley, S. a., Tate, C. G., and Henderson, P. J. (1993) Proton-linked L-rhamnose transport, and its comparison with L-fucose transport in Enterobacteriaceae. Biochem. J. 290 (3), 833-842.

(38) Badía, J., Baldomà, L., Aguilar, J., and Boronat, A. (1989) Identification of the rhaA, rhaB and rhaD gene products from Escherichia coli K-12. FEMS Microbiol. Lett. 53, 253-257.

(39) Grueninger, D., and Schulz, G. E. (2006) Structure and reaction mechanism of L-rhamnulose kinase from Escherichia coli. J. Mol. Biol. 359, 787-797.

(40) Wilson, D. M., and Ajl, S. (1957) Metabolism of L-rhamnose by Escherichia coli. II. The phosphorylation of L-rhamnulose. J. Bacteriol. 73, 415-420.

(41) Kroemer, M., Merkel, I., and Schulz, G. E. (2003) Structure and catalytic mechanism of L-rhamnulose-1-phosphate aldolase. Biochemistry 42, 10560-10568.

(42) Baldomà, L., and Aguilar, J. (1987) Involvement of lactaldehyde dehydrogenase in several metabolic pathways of Escherichia coli K12. J. Biol. Chem. 262, 13991-13996.

(43) Baldomà, L., and Aguilar, J. (1988) Metabolism of L-fucose and L-rhamnose in Escherichia coli: aerobic-anaerobic regulation of Llactaldehyde dissimilation. J. Bacteriol. 170, 416-421.

(44) Monod, J., Cohen-Bazire, G., and Cohn, M. (1951) [The biosynthesis of beta-galactosidase (lactase) in Escherichia coli; the specificity of induction]. Biochim. Biophys. Acta 7, 585-599.

(45) Boos, W., Schaedel, P., and Wallenfels, K. (1967) Untersuchungen zur Induktion der Lac-Enzyme. Eur. J. Biochem. 1, 382-394.

(46) Lederer, T., Kintrup, M., Takahashi, M., Sum, P. E., Ellestad, G. A., and Hillen, W. (1996) Tetracycline analogues affecting binding to Tn10-Encoded Tet repressor trigger the same mechanism of induction. Biochemistry 35, 7439-7446.

(47) Pédelacq, J.-D., Cabantous, S., Tran, T., Terwilliger, T. C., and Waldo, G. S. (2006) Engineering and characterization of a superfolder green fluorescent protein. Nat. Biotechnol. 24, 79-88.

(48) Wegerer, A., Sun, T., and Altenbuchner, J. (2008) Optimization of an E. coli L-rhamnose-inducible expression vector: test of various genetic module combinations. BMC Biotechnol. 8, 2.

(49) Kolin, A., Balasubramaniam, V., Skredenske, J. M., Wickstrum, J. R. and Egan, S. M. (2008) Differences in the mechanism of the allosteric L-rhamnose responses of the AraC/XylS family transcription activators RhaS and RhaR. Mol. Microbiol. 68, 448-461.

(50) Wong, A., Wang, H., Poh, C. L., and Kitney, R. I. (2015) Layering genetic circuits to build a single cell, bacterial half adder. BMC Biol. 13, 40.

(51) Ozbudak, E. M., Thattai, M., Lim, H. N., Shraiman, B. I., and Van Oudenaarden, A. (2004) Multistability in the lactose utilization network of Escherichia coli. Nature 427, 737-740.

(52) Marbach, A., and Bettenbrock, K. (2012) lac operon induction in Escherichia coli: Systematic comparison of IPTG and TMG induction and influence of the transacetylase LacA. J. Biotechnol. 157, 82-88.

(53) Siegele, D. A., and Hu, J. C. (1997) Gene expression from plasmids containing the $\operatorname{araBAD}$ promoter at subsaturating inducer concentrations represents mixed populations. Proc. Natl. Acad. Sci. U. S. A. $94,8168-8172$.

(54) Arpino, J. A. J., Hancock, E. J., Anderson, J., Barahona, M., Stan, G.-B. V., Papachristodoulou, A., and Polizzi, K. (2013) Tuning the dials of Synthetic Biology. Microbiology 159, 1236-1253.

(55) Liu, Z., Yoshihara, A., Kelly, C., Heap, J., Marqvorsen, M. H. S., Jenkinson, S. F., Wormald, M. R., Otero, J. M., Estévez, A., Kato, A., Fleet, G. W. J., Estévez, R. J., and Izumori, K. (2016) 6-Deoxyhexoses from L-Rhamnose in the Search for Inducers of the Rhamnose Operon: Synergy of Chemistry and Biotechnology. Chem. Eur. J., DOI: $10.1002 /$ chem.201602482.

(56) Izumori, K. (2006) Izumoring: a strategy for bioproduction of all hexoses. J. Biotechnol. 124, 717-722.
(57) Gullapalli, P., Yoshihara, A., Morimoto, K., Rao, D., Akimitsu, K., Jenkinson, S. F., Fleet, G. W. J., and Izumori, K. (2010) Conversion of 1-rhamnose into ten of the sixteen 1-and 6-deoxyketohexoses in water with three reagents: d-tagatose-3-epimerase equilibrates C3 epimers of deoxyketoses. Tetrahedron Lett. 51, 895-898.

(58) Shompoosang, S., Yoshihara, A., Uechi, K., Asada, Y., and Morimoto, K. (2014) Enzymatic production of three 6-deoxyaldohexoses from L-rhamnose. Biosci., Biotechnol., Biochem. 78, 317325.

(59) Gibson, D. G., Young, L., Chuang, R. Y., Venter, J. C., Hutchison, C. A., and Smith, H. O. (2009) Enzymatic assembly of DNA molecules up to several hundred kilobases. Nat. Methods 6, 1216.

(60) Davidson, E. A., Basu, A. S., and Bayer, T. S. (2013) Programming microbes using pulse width modulation of optical signals. J. Mol. Biol. 425, 4161-4166.

(61) Salis, H. M., Mirsky, E. A., and Voigt, C. A. (2009) Automated design of synthetic ribosome binding sites to control protein expression. Nat. Biotechnol. 27, 946-950. 